D'après la remarque qui précède, il y a par suite 3 cas possibles, à savoir $2 t+\varepsilon=1,2 t+\varepsilon=16$ et $2 t+\varepsilon \geqslant 34$, qui donnent respectivement $t=0, \varepsilon=1$ et $a=1$, ou bien $t=1, \varepsilon=-1$ et $a=F_{3}-1=2^{8}$, ou bien $t=8, \varepsilon=0$ et $a=8 F_{3}$, ou bien $t \geqslant 17, \varepsilon \geqslant 0$ et $a \geqslant F_{2} F_{3}$, ou enfin $t \geqslant 18$ et $a \geqslant 18 F_{3}-1$. Donc, (2) entraîne pour $a \geqslant 1$ que

(3) $\quad a=1$, ou bien $a=2^{8}$, ou bien $a=8 F_{3}$, ou bien $a \geqslant F_{2} F_{3}$. "

Ceci établi, examinons les $a>1$ naturels assujettis à (1). On a $a=$ $=F_{4} t+\varepsilon$ (où $t \geqslant 1$ et $\varepsilon=0$ ou $\varepsilon= \pm 1$ ) et vu que $F_{4} \equiv 2\left(\bmod F_{1} F_{2} F_{3}\right)$, on conclut que $F_{1} F_{2} F_{3} \mid(2 t+\varepsilon)\left[(2 t+\varepsilon)^{2}-1\right]$.

D'après (3), on a ici $2 t+\varepsilon=1$, ou bien $2 t+\varepsilon=2^{8}$, ou bien $2 t+\varepsilon=8 F_{3}$, ou enfin $2 t+\varepsilon \geqslant F_{2} F_{3}$, ce qui donne 5 cas possibles suivants:

1. $t=1, \varepsilon=-1, a=F_{4}-1=a_{1}$;

2. $t=2^{7}, \varepsilon=0, a=2^{7} F_{4}=a_{2}$;

3. $t=4 F_{3}, \varepsilon=0, a=4 F_{3} F_{4}=a_{3}$;

4. $t=\frac{1}{2}\left(F_{2} F_{3}-1\right), \varepsilon=1, a=\frac{1}{2} F_{4}\left(F_{2} F_{3}-1\right)+1=a_{4}$;

5. $t \geqslant \frac{1}{2}\left(F_{2} F_{3}+1\right), a \geqslant \frac{1}{2} F_{4}\left(F_{2} F_{3}+1\right)-1=a_{5}$.

Or $a_{1}^{2}+1=F_{5}, 13\left|a_{2}^{2}+1,37\right| a_{3}^{2}+1,2 \mid a_{4}^{2}+1$ et $a_{5}=8\left(2^{4}+1\right) \times$ $\times\left(2^{8}+1\right)\left(2^{12}+1\right)$, ce qui achève la démonstration.

Démonstration $d u$ théorème 2. Soient $a$ et $m$ des nombres naturels quelconques dont $a>1$. Il existe par hypothèse un nombre premier de Fermat $F_{i}$ tel que $F_{i} \nmid a\left(a^{2^{2}}-1\right)$. On a $F_{i} \mid a^{F_{i}-1}-1$ en vertu du théorème d'Euler et comme

$$
a^{F_{i}-1}-1=\left(a^{2^{m}}-1\right) \prod_{j=m}^{2^{i}-1}\left(a^{2^{j}}+1\right),
$$

on a $F_{i} \mid a^{2}+1$ pour un $j \geqslant m$.

Si $a^{2^{j}}+1=F_{i}$, il vient $a=2^{2^{i-j}}$, ce qui est incompatible avec l'hypothèse. On a donc $a^{2^{j}}+1 \neq F_{i}$ et le nombre $a^{2^{j}}+1$ (où $j \geqslant m$ ) est composé.

Regu par la Rédaction le 29.1. 1962

\section{REMARK ON RATIONAL TRANSFORMATIONS}

BY

W. NARKIEWICZ (WROCEAW)

In [1] and [2] it was proved that if a field $K$ is finitely generated over the rationals, and $X$ is an infinite subset of $K$, then every polynomial mapping $X$ onto itself must be linear. It seems to be true that every rational function mapping an infinite subset $X$ of such a field onto itself must be a homography. The purpose of this note is to prove this in the case of the field $R$ of rational numbers.

Let $R_{\infty}$ be the set obtained by adjoining an ideal element $\infty$ to $R$. For every rational function $F^{\prime}(t)$ we put $F^{\prime}(\infty)=\lim _{|t| \rightarrow \infty}\left|F^{\prime}(t)\right|$ and if $z$ is a pole of $F^{\prime}(t)$, then we put $F^{\prime}(z)=\infty$. We shall prove the following

THEOREM. If $X$ is an infinite subset of $R_{\infty}$, and $F(t)$ a rational function, such that $X \subset F(X)$, then $F(t)=(a t+b) /(c t+d)$ with suitable rational $a, b, c, d$.

A. Schinzel posed the following problem (see [3]):

Let $f(x, y)$ be a polynomial with rational coefficients, and $X$ an infinite set of rational numbers with the property that for every $x$ in $X$ there exists such an $y$ in $X$ that $f(x, y)=0$. Prove that $f(x, y)$ must have a factor which is linear in $y$ or symmetrical in $x, y$.

As a corollary of our theorem we obtain a positive solution of that problem in the case of $f(x, y)=P(y)-Q(y) x$.

LEMMa 1. Suppose that $X$ is a set and $T$ a transformation mapping a subset $X_{0}$ of $X$ onto $X$. Suppose moreover that there exists a function $s(x)$ defined on $X$ with values in the set of natural numbers subject to conditions:

(i) For every constant $c$ the equation $s(x)=c$ has only a finite number of solutions.

(ii) There exists a constant $C$ such that from $s(x) \geqslant C$ follows $s(T x)$ $>s(x)$.

Then the set $X$ is finite.

Proof of the lemma. If $X=X_{0}$, then the finiteness of $X$ follows from lemma 1 in [1] if we put there $f(x)=s(x), g(x)=1$ for all $x$ and 
$B(M)=2$ for every $M$. Suppose now that $X \backslash X_{0}$ is non-void. Let us associate with every $x$ in $X \backslash X_{0}$ an infinite sequence $y_{1}^{(x)}, y_{2}^{(x)}, \ldots$, and adjoin these sequences to the set $X$ to obtain a set $Y$. Let us define for every $x$ in $X \backslash X_{0}$ and $m=1,2, \ldots: s\left(y_{m}^{(x)}\right)=s(x)+m ; \quad T x=y_{1}^{(x)}$, $T y \underset{m}{(x)}=y_{m+1}^{(x)}$.

Conditions (i) and (ii) are obviously satisfied by the set $Y$ and the extended transformation $T$ and function $s(x)$; moreover $T(Y)=Y$. Hence, as in the case $X=X_{0}$, we can apply lemma 1 of [1] to obtain the finiteness of $Y$, which is clearly a contradiction.

For any polynomial $W(t)$ let us write $W(p, q)=q^{r} W(p / q)$, where $r$ is the degree of $W(t)$.

LEMMA 2. If $P(t), Q(t)$, are relatively prime polynomials with integral coefficients, then the greatest common divisor:

$$
\mu(p, q)=(P(p, q), Q(p, q))
$$

$i s$, for all relatively prime integers $p, q$, bounded by a constant depending on $P$ and $Q$ only, but not on $p, q$.

This lomma is woll-known, but I was not wblo to find a source to quote, and so I give a proof for the convenience of the reader.

Proof. There exist an integer $A$ and polynomials $G(t), H(t)$ with intogral cocfficients such that $P(t) G(t)+Q(t) H(t)=A$. Let $m, n, r, s$ be the degrees of $P, Q, G, H$, respectively. Then, with $k=\max (m+r, n+s)$, $j=k-m-r, j^{\prime}=k-n-s$, we have

$$
A q^{k}=q^{j} P(p, q) G(p, q)+q^{j^{\prime}} Q(p, q) H(p, q) ;
$$

thus $\mu(p, q)$ divides $A q^{k}$. Let $v(p, q)=(\mu(p, q), q)$. It follows immediately that $\nu(p, q)$ divides the coefficient of $t^{m}$ in $P(t)$ and so is bounded by a constant independent of $p, q$.

Let us put $\mu(p, q)=d_{1}(p, q) \cdot v(p, q), q=d_{2}(p, q) v(p, q)$. Then $d_{1}(p, q)$ divides $A \cdot v(p, q)^{k-1}$, whence it is also bounded by a constant independent of $p, q$. Consequently the same may be said about $\mu(p, q)$.

Proof of the theorem. Let $X$ be an infinite subset of $R_{\infty}$ and $F(t)=P(t) / Q(t)$ a rational function such that $X \subset F(X) .(P(t)$ and $Q(t)$ are relatively prime polynomials with integral coefficients, of degree $m, n$ respectively).

Let us define: $T(x)=F(x), \quad s(\infty)=1$ and $s(p / q)=|p|+q$ if $(p, q)=1$ and $q>0$; then put in lemma 1 the set $X$ for $X_{0}$ and $F^{\prime}(X)$ for $X$. Condition (i) is thus obviously satisfied. Now it is sufficient to prove that if $F(t)$ is not a homography then condition (ii) is also satisfied, for in this case lemma 1 would lead to contradiction with the assumption that $X$ is infinite.
Let $w_{1}(p, q)=\left(q^{|n-m|}, P(p, q)\right), w_{2}(p, q)=\left(q^{|n-m|}, Q(p, q)\right)$. It can be easily seen that $w_{1}$ and $w_{2}$ are bounded by a constant independent of $p, q$.

Let $F(p / q)=A \mid B \neq 0 \quad((p, q)=1,(A, B)=1)$. If $n \geqslant m$, then $A \geqslant q^{n-m} P(p, q) / \mu(p, q) w_{2}(p, q)$ and $B \geqslant Q(p, q) / \mu(p, q) w_{2}(p, q)$. Thus in this case

$$
s(F(p / q)) \geqslant\left\{q^{n-m}|P(p, q)|+|Q(p, q)|\right\} / \mu(p, q) w_{2}(p, q) .
$$

Similarly if $n<m$, then

$$
s(F(p / q)) \geqslant\left\{|P(p, q)|+|Q(p, q)| q^{m-n}\right\} \mid \mu(p, q) w_{1}(p, q) .
$$

Lemma 2 leads us to

(1) $s(F(p / q)) \geqslant\left\{\begin{array}{lll}\frac{1}{M_{1}}\left[|P(p, q)|+|Q(p, q)| q^{m-n}\right] & \text { if } \quad m>n, \\ \frac{1}{M_{1}}\left[|P(p, q)| q^{n-m}+|Q(p, q)|\right] & \text { if } \quad m \leqslant n\end{array}\right.$

with some constant $M_{1}>0$.

Lot now $m \geqslant n$. Thon $s(F(p / q)) \geqslant q^{m}\{|P(p / q)|+|Q(p / q)|\} / M_{1}$.

Suppose that for an infinite sequence $p_{k} / q_{k}$ we have $s\left(F\left(p_{k} / q_{k}\right)\right)$ $\leqslant s\left(p_{k} / q_{k}\right)$

We must prove that under this assumption $F(t)$ is a homography. We shall distinguish two cascis: (ఓ) for infinitcly many $k:\left|p_{k}\right| \leqslant W q_{k}$ with some constant $W$, and (b) the sequence $\left|p_{k}\right| q_{k} \mid$ tends to infinity.

In the case (a) we ean freely assume that $\left|p_{k}\right| \leqslant W q_{k}$ holds for every $k$, and then $s\left(p_{k} / q_{k}\right) \leqslant(1+W) q_{k}$. Consequently

$$
q_{k}^{m-1}\left(\left|P\left(p_{k} / q_{k}\right)\right|+\left|Q\left(p_{k} / q_{k}\right)\right|\right) \leqslant(1+W) M_{1} \text {. }
$$

As the polynomials $P(t)$ and $Q(t)$ have no common zeros, thore exists a positive constant $M_{2}$ such that $|P(t)|+|Q(t)| \geqslant M_{2}$ holds for every $t$, and so from (2) we infer $q_{k}^{m-1} \leqslant(1+W) M_{1} / M_{2}$, which is possible for $m=0,1$ only (since $q_{k}$ tends to infinity) and a fortiori for $n=0,1$, but this means that $F(t)$ is a homography.

In the case (b) we obtain

$$
\frac{q_{k}^{m}}{\left|p_{k}\right|}\left(\left|P\left(p_{k} \mid q_{k}\right)\right|+\left|Q\left(p_{k} / q_{k}\right)\right|\right) \leqslant M_{1}\left(1+\left|q_{k} / p_{k}\right|\right) .
$$

At least one of the polynomials $P(t), Q(t)$ is not constant, and as $n \leqslant m$, it is $P(t)$ which is not constant. Consequently for sufficiently 
great $\left|p_{k} / q_{k}\right|$ we have $\left|P\left(p_{k} / q_{k}\right)\right| \geqslant M_{3}\left|p_{k} / q_{k}\right|^{m}$ with a suitable positive $M I_{3}$. It follows that the left side of $(3)$ is at least

$$
H_{3} \cdot \frac{q_{k}^{m}}{\left|p_{k}\right|} \cdot \frac{\left|p_{k}\right|^{m}}{q_{k}^{m}}=M_{3}\left|p_{k}\right|^{m-1},
$$

but the right side of (3) is bounded, and so we infer that $m=0,1$ and a fortiori $n=0,1$, which means that $F(t)$ is a homography. In the case $w>m$ the proof is almost the same, as can be easily seen from the symmetry of (1). We proved thus that if $x \neq \infty, F(x) \neq 0, \infty$ and $s(x)$ is sufficiently great, then $s(F(x))>s(x)$. But in all remaining cases $s(x)$ is bounded by a constant. Consequently if $F(t)$ is not a homography the condition (ii) of lemma 1 is verified, which completes the proof of the theorem.

\section{REFERENCES}

[1] W. Narkiewicz, On polynomial transformations, Acta Arithmetica 7 (1962), p. $241-249$.

[2] - On polynomial transformations II, Acta Arithmetica 8 (1962), p. 11 - 19.

[3] A. Schinzel, $P 417$, this volume, p. 187.

Reçu par la Rédaction le 19.5. 1962
VOL. $\mathrm{X}$ 1963 FASC. 1

ON THE DERIVATIYE OF CLOSE-TO-CONVEX FCNCTIONS

$\mathrm{BY}$

J. KRZYŻ (LUBLIN)

Let $D$ be a simply connected domain of hyperbolic type, i. e. a domain conformally equivalent to an open circle. Then the following definitions of close-to-convexity of $D$ may be considered.

(B): $D$ is said to be close-to-convex, or accessible from outside along rays [1], if the complement of $D$ can be represented as a union of closed rays which do not cross each other.

(K): $D$ is said to be close-to-convex, if for the function $f(z)$ mapping $D$ conformally onto the unit circle $K=\{z:|z|<1\}$ a univalent and convex function $\Phi(z), z \in K$, ean be chosen so that $\mathcal{R}\left\{f^{\prime}(z) / \Phi^{\prime}(z)\right\}>0$ for any $z \in K$ (see [2]).

As pointed out by Lewandowski [3], both definitions of close-to-convexity are equivalent.

For a domain $D$ bounded by a Jordan eurve $\Gamma$ with a continuously changing tangent another equivalent definition of close-to-convexity was given in [2].

$\left(\mathrm{K}_{1}\right): D$ is said to be close-to-convex, if the maximal angle of a clockwise rotation of the outward normal along any subarc of $\Gamma$ described in the positive (counter-clockwise) direction does not surpass $\pi$. Therefore we can also consider close-to-convex curves.

In particular, the class $(L)$ of univalent functions $f(z)=z+a_{2} z^{2}+\ldots$ mapping $K$ onto close-to-convex domains, i. e. the class of close-to-convex functions (introduced independently by Biernacki [1] and Kaplan [2]), may be considered. The class $(L)$ contains functions such as convex, starlike, convex in one direction [5], starlike with respect to symmetric points [6], functions with the derivative of positive real part etc.

In [1], which does not seem to be universally known, Biernacki determined the region of variability of the functionals $\{z / f(z)\},\left\{z f^{\prime}(z) / f(z)\right\}$, for a fixed $z \epsilon K$ and $f$ ranging over $(L)$. In this article we solve an analogous problem for $\log f^{\prime}(z)$ (Theorem 1), and hence we deduce the precise estimates of $\arg f^{\prime}(z)$ for $f \epsilon(L)$ (Theorem 2). In spite of the fact that the 